\title{
Singapore Defibrillation Guidelines 2016
}

Chun Yue Francis Lee ${ }^{1}$, MBBS, FRCS, Venkataraman Anantharaman ${ }^{2}$, MBBS, FRCPEd, Swee Han Lim ${ }^{2}$, FRCSEd, FRCPEd, Yih Yng $\underline{\mathrm{Ng}}^{3}$, MBBS, MPH, Tek Siong $\underline{\mathrm{Chee}}^{4}$, MBBS, MMed, Chong Meng $\underline{\text { Seet }}^{1}$, MBBS, FRCs, Marcus Eng Hock $\underline{\mathrm{Ong}}{ }^{2,5}$, MBBS, MPH

\begin{abstract}
The most common initial rhythm in a sudden cardiac arrest is ventricular fibrillation or pulseless ventricular tachycardia. This is potentially treatable with defibrillation, especially if provided early. However, any delay in defibrillation will result in a decline in survival. Defibrillation requires coordination with the cardiopulmonary resuscitation component for effective resuscitation. These two components, which form the key links in the chain of survival, have to be brought to the cardiac victim in a timely fashion. An effective chain of survival is needed in both the institution and community settings.
\end{abstract}

Keywords: cardiopulmonary resuscitation, defibrillation, defibrillator, public access defibrillation, ventricular fibrillation

\section{INTRODUCTION}

Heart disease is the second leading cause of death in Singapore. It increases the risk of death from sudden cardiac arrest (SCA). The initial rhythm of SCA is most commonly a malignant ventricular arrhythmia, either ventricular fibrillation (VF) or pulseless ventricular tachycardia $(\mathrm{pVT}){ }^{(1,2)}$ the occurrence of which inhibits the activity of the natural pacemakers of the heart, causing cardiac standstill. The key treatment for VF and pVT is defibrillation, supported by good cardiopulmonary resuscitation (CPR). Therefore, defibrillation and CPR remain the cornerstone of both basic and advanced cardiac life support. When delivered early, they confer significant survival benefits. ${ }^{(3,4)}$

\section{APPROACH TO DEFIBRILLATION Waveforms}

Defibrillators are designed to deliver an effective current to the fibrillating heart without causing significant damage to the myocardium or injury to the SCA victim. The traditional monophasic waveform, in which the electric current is delivered to the heart in one direction, has largely been superseded by the biphasic waveform design, which allows for the miniaturisation of defibrillators and reduction of energies required for effective defibrillation. Three common biphasic waveforms, viz. biphasic truncated exponential, rectilinear biphasic and pulsed biphasic, are in clinical use, but there is no major evidence indicating the superiority of one waveform over another. Although biphasic waveforms are most frequently used today, monophasic defibrillation is still effective in the management of VF, and is not more harmful or less successful when compared to biphasic defibrillation.

\section{Defibrillation energy}

Current data suggests that biphasic shocks of either 150 J or 200 J for the first shock convert VF at approximately the same rate. ${ }^{(5)}$ Biphasic shocks of high energy levels (360 J) are potentially harmful if used in low-weight small animals, as demonstrated in animal studies, ${ }^{(6,7)}$ but not in humans. ${ }^{(8,9)}$ Based on these findings, it is reasonable to recommend that, based on the type of waveform used and the manufacturer's recommendations for the model of defibrillator, energy levels in the range of 120-200 J can be used to deliver the first shock for VF. The energy for subsequent shocks can be escalated if the initial energy does not defibrillate the patient and if the defibrillator is capable of providing higher escalating energy levels of up to a maximum of $360 \mathrm{~J}$.

\section{Pads vs. paddles}

Defibrillation using self-adhesive defibrillation pads is becoming increasingly popular, as it is the key interface for delivering electric current in all automated external defibrillators (AEDs) and an alternative to paddles for manual defibrillators. From a practical standpoint, self-adhesive pads confer a number of advantages. When applied correctly, they offer good contact with the skin for delivering shock current, allow for defibrillation to proceed hands-free, provide seamless handover between resuscitation teams if compatible defibrillators are used, are disposable and reduce the risk of cross-infection. Self-adhesive pads are typically applied in the initial steps of resuscitation while chest compression is ongoing.

Paddle defibrillation is the standard option for most manual defibrillators, but application requires more coordination and practice in order to reduce the exposure time of the paddle surface. The paddles need to be either in the defibrillator housing or on the patient. When applying the paddles, the operator is taught to apply pressure to reduce transthoracic impedance. They are applied during the window period for patient and rhythm assessment, after a 1-2-minute cycle of chest compressions, to detect a shockable rhythm. However, during the transfer of the paddles from the housing to the patient's chest, care must be taken to avoid any accidental discharge.

${ }^{1}$ Acute and Emergency Care Centre, Khoo Teck Puat Hospital, ${ }^{2}$ Department of Emergency Medicine, Singapore General Hospital, ${ }^{3}$ Medical Department, Singapore Civil Defence Force, ${ }^{4}$ Chee Heart Specialist Clinic, Parkway East Hospital, ${ }^{5} \mathrm{Health}$ Services and Systems Research, Duke-NUS Medical School, Singapore

Correspondence: Dr Francis Lee Chun Yue, Senior Consultant, Acute and Emergency Care Department, Khoo Teck Puat Hospital, 90 Yishun Central, Singapore 768828. lee.francis@alexandrahealth.com.sg 


\section{Placement of defibrillation electrode (pads or paddles)}

The recommended placement of electrodes is in the sternal-apical position. The right (sternal) electrode is placed to the right of the sternum, below the right clavicle. The apical electrode is placed on the left mid-axillary line at the level of the $\mathrm{V} 6$ electrocardiography electrode. For the layperson, this position is described as the side of the chest below and to the left of the left nipple. There are alternative electrode positions that may be used in situations where the standard position is not possible: (a) bi-axillary position placement of each electrode on the lateral chest walls, one on the right side and the other on the left; (b) apicoposterior position one electrode in the standard apical position and the other on the right upper back; and (c) anteroposterior position - one electrode anteriorly over the left precordium and the other posteriorly to the heart just inferior to the left scapula.

Besides these alternative electrode placement positions, there are also specific precautions to be considered: (a) in women, the electrode should not be placed over breast tissue; (b) in patients with implantable medical devices (e.g. pacemakers, implantable cardioverter defibrillator), the electrode must not be placed directly over these devices; (c) it is recommended that a distance of approximately four-finger breadths should be kept between the electrode/paddle/pad and these devices; (d) drug delivery patches and personal jewellery (especially ones that are metallic in nature) worn over the chest must be removed to avoid potential burns caused by the current coursing over them; and (e) wetness over the chest wall should be cleared with a dry towel before application of the defibrillator pad/paddle.

\section{Manual vs. automated defibrillation}

The key difference between manual and automated defibrillation, apart from the choice of electrode, is the decision process involved in delivering the shock. Manual defibrillation requires the operator to analyse the rhythm through visual examination of the rhythm displayed on a monitor, whereas automatic defibrillation is assisted by an algorithm-based machine analysis and the decision to shock is cued by a voice prompt. Prehospital defibrillation with an AED by non-advanced life support ambulance crew has shortened the time taken for defibrillation. ${ }^{(10,11)}$ This has been shown to improve survival over CPR alone in many studies.

\section{Timing of defibrillation}

With its increasing duration, VF will become refractory to defibrillation, eventually degenerating to asystole, and the probability of survival declines by $7 \%-10 \%$ with every minute of delay in defibrillation. ${ }^{(12)}$ Defibrillation given within three minutes of cardiac arrest can produce a survival rate of as high as $74 \% .{ }^{(13)}$ $\mathrm{CPR}$, when added to the resuscitation process, reduces the rate of decline in survival to as low as $3 \%-4 \%$ for every minute of delay in defibrillation. ${ }^{(12,14)}$

\section{Management of persistent VF}

There are two management options for persistent VF (i.e. VF that fails to convert to another rhythm after defibrillation): single shock followed by CPR or three-stacked shocks. The three-stacked shock strategy is based on the notion that a persistent VF is more amenable to termination when shocks are delivered consecutively. Recent evidence shows that there is no difference in VF recurrence or outcome in terms of survival between the two strategies. ${ }^{(15,16)}$ As in the 2011 National Resuscitation Council guidelines, a single shock followed by CPR is favoured over the three-stacked shock strategy ${ }^{(17)}$ for persistent VF. With biphasic machines, the termination rate of VF is very high. As there is a delay in recovery to a perfusing rhythm immediately after a shock, CPR should be resumed immediately. The single-shock strategy, on the other hand, reduces interruption to CPR.

\section{Asystole vs. fine VF}

During manual defibrillation, an operator may have difficulty discerning an asystole from fine VF displayed on screen. In such a situation, defibrillation should not be attempted. Instead, one should continue CPR and manage the rhythm as for an asystole.

\section{Drug-assisted defibrillation}

Selective medications have been used to increase the success of defibrillation based on the knowledge of their inherent antiarrhythmic activities. Adrenaline is thought to 'coarsen' VF, making it more amendable to defibrillation. The drug, at best, acts to facilitate the restoration of a perfusing rhythm and, by itself, is unlikely to terminate VF/pVT. No drug has been shown to improve outcomes in terms of survival or survival with good neurologic outcome. As such, it is recommended that gaining intravenous access and drug administration should not delay or interfere with CPR or defibrillation. The use of drugs is as recommended in the 2016 Advanced Cardiac Life Support guidelines. ${ }^{(18}$

\section{Defibrillation for shockable rhythms (VF/pVT)}

If a shockable rhythm, such as VF or pVT, is identified during the rhythm check, the defibrillator should be charged immediately. During the time taken to charge the defibrillator, CPR should resume so as to avoid prolonged interruptions to chest compressions, as charging time may vary from 3-15 seconds depending on the model of defibrillator.

The energy should be set at $360 \mathrm{~J}$ for monophasic defibrillators and 120-360 J for biphasic defibrillators, depending on the manufacturer and model. Once charged, CPR is interrupted to clear the patient only long enough to deliver the shock safely, within ten seconds. Self-adhesive defibrillator pads are as effective as paddles for defibrillation, and are preferred for safety and ease of use. They should be applied with minimal or no interruptions to CPR. Paddles are acceptable as backups in the event that pads are unavailable, but they should be handled safely. Healthcare professionals should remain familiar and competent in the use of both pads and paddles.

Following each shock, there is no benefit in re-analysing the rhythm, whether manually or with the AED. Chest compressions should be resumed immediately after each shock, for 1-2 minutes until the next electrocardiogram analysis. Interrupting chest compressions to analyse the rhythm or check for a pulse immediately after the delivery of a shock will prolong the 
peri-shock pause. Prolonged peri-shock pauses are associated with poorer outcomes. A pulse could have returned at any time during the cycle of 1-2 minutes of CPR, during which chest compressions are ongoing, without harm to the patient. Escalating shock energy may be considered for refractory VF. Stacks of three shocks are not currently recommended in routine practice.

\section{COORDINATION OF DEFIBRILLATION WITH CPR \\ CPR before defibrillation}

Current evidence from studies on out-of-hospital cardiac arrest (OHCA) indicates that a period of CPR before defibrillation does not confer any survival benefits. Therefore, in the initial approach to a cardiac arrest, CPR should be started immediately. Once the defibrillator is available, defibrillation should be carried out without further delay, if indicated.

\section{CPR-defibrillation sequence}

In advanced life support, high-quality CPR and timely defibrillation are both essential in the chain of survival. Although CPR should be carried out with minimal interruptions, it should not cause a delay in initiation of defibrillation, as success in treating a shockable rhythm is reduced even with a 5-10-second delay. To address the opposing needs of these two strategies, the following steps are recommended to ensure timely defibrillation with minimal pre-shock pause to CPR:

- $\quad$ Once the defibrillator is available, a 'quick look' is carried out, either with adhesive monitoring pads or defibrillation paddles, to assess the heart rhythm.

- If VF or pVT is noted, CPR should be immediately resumed while the defibrillator is charging up.

- A shock is immediately given once the machine is ready to shock. When attempting a shock, all personnel must 'stand clear' of the patient.

- $\quad$ Once the shock is delivered, CPR is resumed. There should not be attempts to check the rhythm, pulse or breathing at this juncture, as this can delay continuation of chest compressions.

- $\quad$ The next rhythm (and/or pulse and breathing) is checked after 1-2 minutes of CPR.

The immediate resumption of CPR after defibrillation is very important, as it has been shown that some patients may remain pulseless for up to two minutes and up to $25 \%$ of these patients may have return of spontaneous circulation beyond two minutes. Moreover, post-shock CPR does not cause harm or lead to the recurrence of VF.

\section{DEFIBRILLATION SAFETY} Hands-on defibrillation

This refers to the continuation of CPR while defibrillation is taking place, with the aim of reducing peri-shock interruption in chest compressions. While continuation of CPR during defibrillation reduces hands-off time, there have been concerns about the potential harm of electrical shocks to the CPR provider. It has been suggested that this procedure may be safely carried out through the use of double-layered polyethylene gloves or special electrical safety gloves. ${ }^{(19-21)}$ Team training and preparations are required to incorporate this procedure into the resuscitation workflow.

\section{Oxygen use and defibrillation}

In an oxygen-rich environment, electrical discharge from defibrillation could potentially pose fire and burn hazards. To minimise these risks, the following recommendations are given (unchanged from the 2011 Singapore NRC guidelines): ${ }^{(17)}$ as far possible, oxygen sources should be maintained in a closed circuit (e.g. intubated patients); and open oxygen sources should be located a reasonable distance away from the site of defibrillation (e.g. nasal cannula or oxygen masks should be moved away from the chest wall or their oxygen source turned off prior to defibrillation). During defibrillation, properly applied self-adhesive pads and well-placed paddles would minimise the risk of electrical arcing. ${ }^{(22)}$

\section{Fully automated external defibrillator}

Fully automated defibrillators (i.e. defibrillators that can detect VF and deliver a shock without human intervention) are deemed safe for use based on studies on defibrillation training and simulated scenarios. However, evidence of safe application on humans is lacking. The replacement of current AEDs with fully automated defibrillators is not currently recommended. ${ }^{(23)}$

\section{Defibrillation in the hospital setting}

There were no good trials to compare manual defibrillation with AED use in the hospital setting. There are, however, two trials that showed that the application of AEDs, supplementing manual defibrillation use, in an organised AED programme, led to higher survival to hospital discharge for in-hospital patients with shockable rhythms. ${ }^{(24,25)}$ Studies that looked at the application of AEDs vs. no AED use in patients that suffered in-hospital cardiac arrest (IHCA) have shown reduced survival to discharge rate for non-shockable rhythms or equivalent rates in shockable rhythms. In these circumstances, slow AED use could have led to delays in starting CPR or interruptions to CPR during rhythm check. ${ }^{(26)}$ Recommendations for AED use in IHCA are as follows:

- $\quad$ AED should be deployed in areas of the hospital where there is a risk of delay to defibrillation. These include areas that are more easily accessed by members of the public or non-medical personnel and situations in which healthcare providers are not trained to deliver manual defibrillation despite their immediate availability.

- $\quad$ AED use should be part of the established chain of survival, with the aim of delivering defibrillation within three minutes of cardiac arrest. Where personnel who could have delivered manual defibrillation are present, manual defibrillation is preferred over AED.

\section{PUBLIC ACCESS DEFIBRILLATION An effective strategy to manage OHCA}

The review of public access defibrillation (PAD) strategy by the International Liaison Committee on Resuscitation has rated public access defibrillation as a Class I recommendation 
(Class I, LOE C-LD). ${ }^{(27-29)}$ The data is derived mainly from registry studies and one randomised controlled trial, which showed that 30 day survival improves with a PAD programme, as compared with not having one. Data from large registry studies in Japan and the USA have also shown that when AEDs are available, defibrillation occurs sooner and the odds of survival are increased. First responder and PAD programmes are proven ways to systematically raise OHCA survival. ${ }^{(13,30)}$

\section{Safe use of PAD by laypersons}

The European Resuscitation Council 2010 guidelines highlighted eight studies in a systematic review that had reported a total of 29 PAD-related adverse events, one of which occurred after 1997. ${ }^{(26,31,32)}$ The improvement in defibrillator design interfaces and technology may account for the reduction in adverse events after 1997, even as the use of AEDs increases around the world. All major manufacturers of AEDs today allow the use of AEDs even by untrained responders. However, training is always recommended to improve the speed of response, although the lack thereof should not stop a bystander from attempting to use an AED by following the instructions from the device. Most bystander responders have reported positive experiences from their attempts at intervention. Adverse psychological effects among responders, though rarely noted, should be managed if they arise. ${ }^{(33)}$ Ideally, a surveillance programme should be implemented to record bystander details so as to debrief and offer them psychological assistance. This would enhance data collection, which is necessary to strengthen the chain of survival.

\section{FIRST RESPONDER PROGRAMME IN ORGANISATIONS}

Establishing a first responder programme and planned AED deployment within organisations and uniformed groups is an effective way to increase AED coverage in the public domain and to promote the use of PADs.

\section{Ambulance crew}

It has been amply demonstrated that emergency ambulance crew have saved lives due to defibrillator use. As a result, AED deployment within ambulances has become a standard of care. This deployment and training of AED skills should also be extended to non-emergency ambulances.

\section{Motorcycle-based paramedics}

In large urban communities, such as Singapore, traffic is a key obstacle to timely ambulance access to cardiac arrest victims. Singapore has introduced motorcycle-based paramedics with the primary purpose of rapidly navigating busy roads and reaching patients, cutting down significant minutes in providing AED access.

\section{Aircrafts and airports}

Airports have a high volume of human traffic and see a significant number of cardiac arrest cases. Cardiac arrests could occur in airport premises as well as aircrafts during flight. Many airlines and airports have established chain of survival programmes for cardiac arrest and generally follow these recommendations:

- Establishment of CPR and defibrillation protocols that are certified by a registered medical practitioner.

- All aircrew should be trained to recognise a cardiac arrest, perform CPR and use the AED. In addition, a sizable number of general airport staff should also be trained to ensure adequate airport coverage.

- $\quad$ All aircrafts should carry at least one AED in an easily accessible area of the plane that is known to all aircrew. The time to reach an AED and return to the patient should not exceed three minutes.

- $\quad$ Protocols on how CPR and AEDs are to be conducted within an aircraft environment (e.g. location, safety and methods) should be in place.

- $\quad$ All defibrillation performed on board an aircraft should be clearly documented. The documentation must be reviewed by an authorised medical service for the purpose of audit.

- $\quad$ There should be a system of maintenance of AEDs that are kept on board an aircraft. Such a system must be verified by an authorised medical practitioner.

\section{Medical establishments}

It is a known fact that the first witness of a cardiac arrest situation in a hospital or clinic is often not a doctor. To reduce delays in activation of CPR and AED, medical establishments that provide patient care should install first responder programmes to supplement the standard physician-dependent chain of survival (e.g. Code Blue) protocols:

- $\quad$ All medical and nursing staff in ward and ambulatory areas should be trained and currently competent in the performance of CPR and the use of an AED and/or manual defibrillator.

- Defibrillators should be strategically placed throughout the hospital/medical establishment, close to areas where patients are likely to require resuscitation services.

- In public areas within medical establishments, AEDs should be strategically deployed. Encouraging non-medical staff to be trained in CPR and AED skills will complement the benefits of such AED placement.

\section{Police officers on patrol in cars/motorcycles}

Most communities have more police patrol cars and motorcycles than ambulances. It appears reasonable that a police officer armed with an AED would reach a collapsed patient in the out-of-hospital environment faster than an ambulance-based responder. In communities that have invested in implementing an AED programme in police cars, police officers have contributed to improved survival from OHCA. ${ }^{(34)}$ If the police force is able to respond at least as quickly and confidently as other trained first responders, police officers would have a greater chance of making a significant impact on survival from OHCA.

\section{Firefighters}

In some communities, firefighters often arrive early at the scene of a cardiac arrest and are in a good position to render help 
to cardiac arrest victims. Moreover, CPR and AED skills are complementary to their core responsibilities. Therefore, fire vehicles should be equipped with AEDs for use by fire crew who respond to such incidents.

\section{Security personnel}

Some of the highest survival rates in the world after collapse from VF have been in the casinos of Las Vegas, where AED-trained security offices have achieved survival rates of close to $80 \%$, with collapse-to-first-shock times of under three minutes in most instances. ${ }^{(13)}$ These results indicate that security officers of large establishments are eminently trainable in this skill.

\section{Medical and paramedical personnel of military establishments}

SCA has been noted in various military establishments over the years. The potential for saving such relatively young members of our community would be enhanced by the ready availability of AEDs in such establishments and regular training of their staff in CPR and AED skills.

\section{Designated public first-aiders}

All members of the public are potentially trainable in automated defibrillation to help cardiac arrest victims. In public areas, buildings, work areas, schools and recreational areas such as sports complexes and golf courses, the availability of AEDs and the ability of community citizens to use them can be a boost to survival from OHCA.

\section{RECOMMENDATIONS FOR AED SITING}

In general, public places with at-risk people, large populations, cardiovascular risk activities and limited/delayed access to emergency medical services should be considered for AED installation. ${ }^{(35-37)}$ Rapid first response programmes where AEDs are readily available have reported high survival rates of $49 \%-74 \%$. ${ }^{(38)}$

However, it has been noted that in Singapore, $70 \%$ of OHCA happens in the home. ${ }^{(39)}$ In most countries, PAD programmes are focused only in public places due to the cost-effectiveness of placing AEDs where the risk is concentrated (owing to multiple factors such as high population density, cardiovascular risk activities and number of people with chronic diseases). However, the acquisition of AEDs by individuals for home usage has not been shown to be effective, even for those with high risk of SCA. ${ }^{(40)}$

Singapore has a unique opportunity to improve access to PADs for OHCA occurring in the home, given that over $80.0 \%$ of the population live in clustered public housing with high population density and $14.4 \%$ live in high-rise condominiums. ${ }^{(41)}$ AEDs have been made available in selected neighbourhoods in Singapore over the last eight years. Recently, the Ministry of Home Affairs and Ministry of Health embarked on the installation of AEDs at public housing apartment blocks on a large scale as part of the Save-A-Life initiative, which aims to reach every other public housing block by 2019. ${ }^{(42)}$ In addition, a public awareness initiative known as the Dispatcher Assisted first REsponder (DARE) programme, which features dispatcher-assisted chest compressions and the use of an AED, has been rolled out. A first responder geolocation application called myResponder has also been in operation since June 2015. This app alerts the public emergency ambulance call centre of the location of a cardiac arrest and mobilises any volunteer first responder within $400 \mathrm{~m}$ of the incident. The application also shows the location of the nearest AED. These initiatives may help to address the ability of PAD programmes to improve OHCA survival in residential areas.

\section{ENSURING EFFECTIVENESS OF PAD PROGRAMME}

Data from the United States Centers for Disease Control and Prevention's CARES (Cardiac Arrest Registry to Enhance Survival) programme showed that only $3.7 \%$ of VF arrests had bystander AED applied. ${ }^{(43)}$ In Singapore, the use of PADs was reported to be only $1 \% .{ }^{(39)}$ Clearly, more can be done to increase PAD usage. Kitamura et al noted a relationship among increasing density of AED coverage, improved response times and survival. ${ }^{(44)} \mathrm{A}$ local study $^{(45)}$ found that port/airport/immigration checkpoints, hotels/ hostels, transport facilities, sports/recreational facilities, and health/medical care facilities are high-yield public sites for AED placement. A registry for AED integration, R-AEDi, has recently been established to help link first responders to the nearest AED during an emergency. ${ }^{(46)}$ Apart from increasing the density of installation, other potential factors that may affect the success of a PAD programme include legislation, public education, CPR training, registration, standardisation, operational integration and onsite orientation drills.

\section{REFERENCES}

1. Engdahl J, Holmberg M, Karlson BW, Luepker R, Herlitz J. The epidemiology of out-of-hospital 'sudden' cardiac arrest. Resuscitation 2002; 52:235-45.

2. Rea TD, Eisenberg MS, Becker LJ, Murray JA, Hearne T. Temporal trends in sudden cardiac arrest - a 25-year emergency medical services perspective. Circulation 2003; 107:2780-5

3. Weisfeldt ML, Sitlani CM, Ornato JP, et al; ROC Investigators. Survival after application of automatic external defibrillators before arrival of the emergency medical system: evaluation in the resuscitation outcomes consortium population of 21 million. J Am Coll Cardiol 2010; 55:1713-20.

4. Berdowski J, Blom MT, Bardai A, et al. Impact of onsite or dispatched automated external defibrillator use on survival after out-of hospital cardiac arrest. Circulation 2011; 124:2225-32.

5. Morrison LJ, Henry RM, Ku V, et al. Single-shock defibrillation success in adult cardiac arrest: a systematic review. Resuscitation 2013; 84:1480-6.

6. Berg RA, Samson RA, Berg MD, et al. Better outcome after pediatric defibrillation dosage than adult dosage in a swine model of pediatric ventricular fibrillation. J Am Coll Cardiol 2005; 45:786-9.

7. Xie J, Weil MH, Sun S, et al. High-energy defibrillation increases the severity of postresuscitation myocardial dysfunction. Circulation 1997; 96:683-8.

8. Stiell IG, Walker RG, Nesbitt LP, et al. BIPHASIC Trial: a randomized comparison of fixed lower versus escalating higher energy levels for defibrillation in out-ofhospital cardiac arrest. Circulation 2007; 115:1511-7.

9. Walcott GP, Melnick SB, Killingsworth CR, Ideker RE. Comparison of low energy versus high energy biphasic defibrillation shocks following prolonged ventricular fibrillation. Prehosp Emerg Care 2010; 14:62-70.

10. Weisfeldt ML, Sitlani CM, Ornato JP, et al; ROC Investigators. Survival after application of automatic external defibrillators before arrival of the emergency medical system: evaluation in the resuscitation outcomes consortium population of 21 million. J Am Coll Cardiol 2010; 55:1713-20.

11. Berdowski J, Blom MT, Bardai A, et al. Impact of onsite or dispatched automated external defibrillator use on survival after out-of hospital cardiac arrest. Circulation 2011; 124:2225-32.

12. Larsen MP, Eisenberg MS, Cummins RO, et al. Predicting survival from out-ofhospital cardiac arrest: a graphic model. Ann Emerg Med 1993; 22:1652-8.

13. Valenzuela TD, Roe DJ, Nichol G, et al. Outcome of rapid defibrillation by security officers after cardiac arrest in casinos. N Engl J Med 2000; 343:1206-9. 
14. Valenzuela TD, Roe DJ, Cretin S, Spaite DW, Larsen MP. Estimating effectiveness of cardiac arrest interventions: a logistic regression survival model. Circulation 1997; 96:3308-13.

15. Jost D, Degrange H, Verret C, et al; DEFI 2005 Work Group. DEFI 2005: randomized controlled trial of the effect of automated external defibrillator cardiopulmonary resuscitation protocol on outcome from out-of-hospital cardiac arrest. Circulation 2010; 121:1614-22.

16. Berdowski J, ten Haaf M, Tijssen JG, Chapman FW, Koster RW. Time in recurrent ventricular fibrillation and survival after out-of-hospital cardiac arrest. Circulation 2010; 122:1101-8.

17. Lee F. Defibrillation. Singapore Med J 2011; 52:544-7.

18. Ching CK, Leong SH, Chua SJ, et al; National Resuscitation Council Singapore. Advanced Cardiac Life Support: 2016 Singapore Guidelines. Singapore Med J 2017; 58:360-72.

19. Neumann T, Gruenwald M, Lauenstein C, et al. Hands-on defibrillation has the potential to improve the quality of cardiopulmonary resuscitation and is safe for rescuers-a preclinical study. J Am Heart Assoc 2012; 1:e001313.

20. Deaken CD, Thomsen JE, Løfgren B, Petley GW. Achieving safe hands-on defibrillation using electrical safety gloves--a clinical evaluation. Resuscitation 2015: 90:163-7.

21. Kerber RE. Hands-on defibrillation: "Gloves as sweet as damask roses" (William Shakespeare: the winter's tale). Resuscitation 2015; 90:A6-7.

22. Deakin CD, Nolan JP, Sunde K, Koster RW. European Resuscitation Counci Guidelines for Resuscitation 2010 Section 3. Electrical therapies: automatic external defibrillators, defibrillation, cardioversion and pacing. Resuscitation 2010; 81:1293-304.

23. Hosmans TP, Maquoi I, Vogels C, Courtois AC, et al. Safety of fully automatic external defibrillation by untrained lay rescuers in the presence of a bystander. Resuscitation 2008; 77:216-9.

24. Zafari AM, Zarter SK, Heggen V, et al. A program encouraging early defibrillation results in improved in-hospital resuscitation efficacy. J Am Coll Cardiol 2004 44:846-52.

25. Destro A, Marzaloni M, Sermasi S, Rossi F. Automatic external defibrillators in the hospital as well? Resuscitation 1996; 31:39-43.

26. Gibbison B, Soar J. Automated external defibrillator use for in-hospital cardiac arrest is not associated with improved survival. Evid Based Med 2011; 16:95-6.

27. Hallstrom AP, Ornato JP, Weisfeldt M, et al; Public Access Defibrillation Tria Investigators. Public-access defibrillation and survival after out-of-hospital cardiac arrest. N Engl J Med 2004; 351:637-46.

28. Perkins GD, Travers AH, Berg RA, et al; Basic Life Support Chapter Collaborators Part 3: adult basic life support and automated external defibrillation: 2015 International Consensus on Cardiopulmonary Resuscitation and Emergency Cardiovascular Care Science with Treatment Recommendations. Resuscitation 2015; 95:e43-69.

29. Steill IG, Wells GA, Field BJ, et al. Improved out-of-hospital cardiac arrest survival through the inexpensive optimization of an existing defibrillation program: OPALS study phase II. Ontario Prehospital Advanced Life Support. JAMA 1999; 281:1175-81.

30. Powell J, Van Ottingham L, Schron E. Public defibrillation: increased survival from a structured response system. J Cardiovasc Nurs 2004; 19:384-9.

31. Hoke RS, Heinroth K, Trappe HJ, Werdan K. Is external defibrillation an electric threat for bystanders? Resuscitation 2009; 80:395-401.

32. Dickinson $\mathrm{CL}$, Hall $\mathrm{CR}$, Soar J. Accidental shock to rescuer during successful defibrillation of ventricular fibrillation--a case of human involuntary automaticity. Resuscitation 2008; 76:489.

33. Peberdy MA, Ottingham LV, Groh WJ, et all; PAD Investigators. Adverse events associated with lay emergency response programs: the public access defibrillation trial experience. Resuscitation 2006; 70:59-65.

34. Joglar JA, Page RL. Automated external defibrillator use by police responders: where do we go from here? Circulation 2002; 106:1030-3

35. Nichol G, Valenzuela T, Roe D, Clark L, Huszti E, Wells GA. Cost effectiveness of defibrillation by targeted responders in public settings. Circulation 2003; 108:697-703.

36. Nichol G, Huszti E, Birnbaum A, et al. Cost-effectiveness of lay responder defibrillation for out-of-hospital cardiac arrest. Ann Emerg Med 2009; 54:22635.e1-2.

37. Folke F, Lippert FK, Nielsen SL, et al. Location of cardiac arrest in a city center: strategic placement of automated external defibrillators in public locations. Circulation 2009; 120:510-7.

38. White RD, Bunch TJ, Hankins DG. Evolution of a community-wide early defibrillation programme experience over 13 years using police/fire personnel and paramedics as responders. Resuscitation 2005; 65:279-83.

39. Eng Hock Ong M, Chan YH, Anantharaman V, et al. Cardiac arrest and resuscitation epidemiology in Singapore (CARE I study). Prehosp Emerg Care 2003; 7:427-33.

40. Weisfeldt ML, Everson-Stewart S, Sitlani C, et al; Resuscitation Outcomes Consortium Investigators. Ventricular tachyarrhythmias after cardiac arrest in public versus at home. N Engl J Med 2011; 364:313-21.

41. Department of Statistics, Singapore. Singapore Population Statistics 2016 [online]. Available at: http://www.singstat.gov.sg/statistics/latest-data\#22. Accessed May 27, 2017

42. Ministry of Home Affairs. The SCDF Workplan Seminar 2016 - Speech by Mr K Shanmugam, Minister for Home Affairs and Minister for Law [online]. Available at: https://www.mha.gov.sg/Newsroom/speeches/Pages/The-SCDF-WorkplanSeminar-2016-Speech-by-Mr-K-Shanmugam,-Minister-for-Home-Affairs-andMinister-for-Law.aspx. Accessed May 27, 2017.

43. McNally B, Robb R, Mehta M, et al. Out-of-hospital cardiac arrest surveillance --Cardiac Arrest Registry to Enhance Survival (CARES), United States, October 1 , 2005--December 31, 2010. MMWR Surveill Summ 2011; 60:1-19.

44. Kitamura T, Iwami T, Kawamura T, et al; Implementation Working Group for the All-Japan Utstein Registry of the Fire and Disaster Management Agency. Nationwide public-access defibrillation in Japan. N Engl J Med 2010; 362:994-1004.

45. Zakaria ND, Ong ME, Gan HN, et al; PAROS study group. Implications for public access defibrillation placement by non-traumatic out-of-hospital cardiac arrest occurrence in Singapore. Emerg Med Australas 2014; 26:229-36.

46. R-AEDI: The Registry for Automatic External Defibrillator Integration (2015) [online]. Available at: https://www.raedi.sg/. Accessed May 27, 2017. 